\title{
Simvastatin inhibits the apoptosis of hippocampal cells in a mouse model of Alzheimer's disease
}

\author{
XIAOQIN HU ${ }^{1}$, CHENGWEI SONG $^{2}$, MING FANG $^{2}$ and CHENGYAN LI ${ }^{1}$ \\ ${ }^{1}$ Department of Neurology, Remnin Hospital of Wuhan University, Wuhan, Hubei 430060; \\ ${ }^{2}$ Department of Neurology, The First Hospital of Yichang, The Gorges University \\ College of Medicine, Yichang, Hubei 443000, P.R. China
}

Received May 15, 2016; Accepted March 24, 2017

DOI: $10.3892 / \mathrm{etm} .2017 .5620$

\begin{abstract}
Alzheimer's disease is associated with cognitive impairments that affect memory and executive functions. Simvastatin is a cholesterol-lowering statin drug that is used to control levels of cholesterol in the blood, particularly in cases of hypercholesterolemia, and may be used in the treatment of aneurysmal subarachnoid hemorrhage. Previous results have indicated that the apoptosis of hippocampal cells may serve a critical role in the progression of Alzheimer's disease. In the present study, it was determined whether Simvastatin inhibited the apoptosis of hippocampal cells in vitro and in vivo. The therapeutic effects of Simvastatin were evaluated in 24-month-old triple-transgenic Alzheimer's disease (3xTg-AD) mice, and the efficacy of Simvastatin in attenuating memory and cognitive impairment was investigated. Levels of apoptosis-related gene expression in the hippocampus and hippocampal cells of experimental mice were also detected. In addition, neuron excitability was assessed in the functionally relevant brain regions in the hippocampus. The data indicated that Simvastatin significantly suppressed the apoptosis of hippocampal cells in 3xTg-AD model mice compared with controls $(\mathrm{P}<0.01)$. Furthermore, treatment with Simvastatin improved the dementia status of $3 \times \mathrm{Tg}-\mathrm{AD}$ mice, as determined by a learning task in which mice exhibited significantly reduced attention impairment, impulsivity and compulsivity $(\mathrm{P}<0.01)$. In addition, results demonstrated that Simvastatin significantly inhibited hippocampal damage and significantly improved neuronal loss in hippocampal structures classically associated with attentional performance when compared with untreated mice $(\mathrm{P}<0.01)$. Thus, Simvastatin prevented cognitive impairment by decreasing hippocampal cell apoptosis and improving learning-memory ability. Simvastatin treatment
\end{abstract}

Correspondence to: Professor Chengyan Li, Department of Neurology, Remnin Hospital of Wuhan University, 238 Jiefang Road, Wuhan, Hubei 430060, P.R. China

E-mail: haiyanliulinyi@163.com

Key words: Alzheimer's disease, simvastatin, hippocampal cells, apoptosis also increased the expression of anti-apoptotic genes and decreased the expression pro-apoptotic genes $(\mathrm{P}<0.01)$, which may have been associated with improved motor attention and cognitive competence in 3xTg-AD mice. Collectively, these preclinical data indicated that Simvastatin was efficient in attenuating memory lapse and hippocampal cell apoptosis in a 3xTg-AD mouse model. Thus, Simvastatin may be useful in improving the clinical outcome of patients with Alzheimer's disease.

\section{Introduction}

Alzheimer's disease is a progressive neurodegenerative disorder with a complex etiology, and belongs to a wider class of common memory disorders (1). Previous studies have suggested that Alzheimer's disease is directly associated with hippocampal cells (2-4), and links between neural apoptosis in the corticocerebral and hippocampal regions have been observed in clinical and model studies of Alzheimer's disease $(5,6)$. Previous studies have also indicated that the process of apoptosis serves a key role in the depletion of hippocampal cells and contributes to impairments in attention and cognitive competence through the regulation of various apoptotic factors, including B-cell lymphoma 2 (Bcl-2), Bcl-2-associated death promoter, Bcl-2-associated $\mathrm{X}$ protein (Bax), amyloid precursor protein intracellular C-terminal domain, caspases, tumor necrosis factor-alpha, amyloid beta (Abeta), enzyme activity and reactive oxygen species production, leading to deleterious neurodegenerative disorders such as Alzheimer's disease (7,8). In addition, Bartolomé et al (9) documented that the inhibition of neuronal apoptosis attenuated the phosphorylation of tau and protected memory function, which was beneficial in the treatment of an animal model of Alzheimer's disease. Absalon et al (10) also suggested that activation of cell cycle progression, tau phosphorylation and inhibition of apoptosis in post-mitotic neurons through microRNA regulation contributed to the recovery of mice with Alzheimer's disease. Collectively, these previous data suggest that apoptosis is associated with the complex etiology, pathogenesis and progression of Alzheimer's disease. Based on these findings, it has been suggested that targeting of hippocampal signaling pathways may be a viable therapeutic strategy in the treatment of Alzheimer's disease (11). 
Apoptosis is a fundamental biological process that occurs within cells and is controlled by gene expression. Bcl-2 and Caspase-3 are two key members of the apoptotic family of proteins, and their functions in regulating the apoptosis of brain cells have been widely documented (12-15). A previous study observed that downregulation in Bcl-2 and upregulation in Bax activated P53 and Caspase-3, which lead to the induction of apoptosis in the hippocampal cells of rats (16). Bcl-2 and Caspase-3 are important molecular regulators of apoptosis, and overexpression of Bcl-2 has been demonstrated to inhibit the activation of caspase- 3 and other pro-apoptotic factors (17). Activation of the p38 mitogen-activated protein kinase (MAPK) signaling pathway may mediate hippocampal apoptosis through the regulation of Bcl-2, Bax and Caspase-3. More recently, a number of studies have demonstrated that the expression of $\mathrm{Bcl}-2$ is related to cell survival, and increased expression of Bcl-2 in the brain may reduce cerebral infarct size and protect neural cells (18-21). Furthermore, overexpression of Bcl-2 has been demonstrated to inhibit the activation of Caspase-3, as an initiating factor of apoptosis, which resulted in the suppression of apoptosis in hippocampal cells (22).

Simvastatin is considered to exert beneficial effects in patients with Alzheimer's disease due to its antineoplastic and anti-apoptotic effects in hippocampal cells and axoneurons (23). In patients with Alzheimer's disease, Simvastatin has been documented to overcome the resistance to serum withdrawal-induced apoptosis of lymphocytes (9). Previous work has proposed a novel mechanism for the neuroprotective effects of Simvastatin that involves the modulation of seladin-1, as a metabolic regulator in Alzheimer's disease (24). Furthermore, Simvastatin inhibited the aggregation of Abeta into extracellular cortical and hippocampal plaques in Alzheimer's disease, suggesting that regulation of brain cholesterol may attenuate the pathology of Alzheimer's disease (25). Collectively, these previous data indicate that Simvastatin may be useful as a drug therapy for Alzheimer's disease.

In the present study, it was hypothesized that Simvastatin may exert neuroprotective effects in Alzheimer's disease by regulating the apoptosis of hippocampal neurons. A mouse model of Alzheimer's disease was used to evaluate the therapeutic effects of Simvastatin on cognitive impairment in terms of hippocampal apoptosis and learning-memory ability. Although positron emission tomography (PET) scans in mice with Alzheimer's disease have indicated that hippocampal atrophy is associated with the progression to Alzheimer's disease (26), changes in hippocampal signaling are not well understood. In the current study, 30 mice with Alzheimer's disease were evaluated over a 36-month period through cognitive and serological assessments to determine the efficacy of Simvastatin as a neuroprotective agent. Further preclinical studies are now required to elucidate the full efficacy and tolerability of Simvastatin in the treatment of Alzheimer's disease.

\section{Materials and methods}

Ethical approval. The present study was approved by the Ethics Committee of the People's Hospital of Wuhan University (reference no. 11/H1011/102; Wuhan, China). All surgical and euthanasia (via decapitation) procedures conducted on experimental mice were performed under sodium pentobarbital anesthesia (40 mg/kg; Sigma-Aldrich; Merk KGaA; Darmstadt, Germany) to minimize suffering.

Animal treatment. A total of 60 male triple-transgenic Alzheimer's disease (3xTg-AD mice; weight, 30-35 g; age, 4-6 weeks old) were purchased from the Institute of Biophysics at the Chinese Academy of Sciences (Beijing, China). Mice were divided into two groups [Simvastatin and phosphate-buffered saline (PBS) control, $n=30$ in each group). Mice were treated with a daily intravenous injection of $100 \mathrm{mg} / \mathrm{kg}$ Simvastatin or PBS for 28 days. All mice were housed at preference temperature $\left(22-24^{\circ} \mathrm{C}\right)$ under a 12 -h light-dark cycle and fed ad libitum. Mouse experiments were carried out according to protocols approved by the Institutional Animal Care and Use Committee of the Provincial Hospital affiliated to Shandong University (Jinan, China).

Cognitive and behavioral tests. Following the 28-day treatment period with Simvastatin, the cognitive competence of mice was determined by evaluating their open field activity levels in a black Plexiglas box $(60 \times 60 \times 25 \mathrm{~cm})$. Mice were placed in the open black box for $10 \mathrm{~min}$ and behaviors of the mice were monitored and evaluated with an auto-tracking system (Version 3.2; SmarTrack; Smart Solutions, Inc. Madison, WI, USA) under conditions described previously (27). A Morris water maze test was also performed before and after treatment with Simvastatin to measure cognitive function. The facility used for the Morris water maze experiment was a circular stainless-steel tank (155 cm in diameter, $60 \mathrm{~cm}$ in depth) filled with water to a depth of $40 \mathrm{~cm}\left(27.0 \pm 1.0^{\circ} \mathrm{C}\right)$, which was made opaque by the addition of skimmed milk. For this task, mice were challenged with learning the location of a hidden circular platform $(10 \mathrm{~cm}$ in diameter, $1.5 \mathrm{~cm}$ below the surface of the water) in a fixed location in one quadrant of the tank as described previously (28). To evaluate the functional rehabilitation of the mice treated with Simvastatin and PBS, Rankin scoring was used (29). Mice were kept alive for 36 months to assess the effects of the treatments on survival. The survival rate was monitored every 3 months.

Fluorodeoxyglucose (FDG)-PET imaging. FDG-PET was used to analyze action potential discharge sites and identify regions of cerebral neurons using statistical parametric mapping software (SPM; version 2; The MathWorks, Inc., Natick, MA, USA) prior to and post-treatment with Simvastatin. FDG-PET images were spatially normalized onto the Montreal Neurological Institute (McGill University, Montreal, Canada) PET brain template to define regions of interest. Normalized images were smoothed by convolution with a $10-\mathrm{mm}$ full width at half maximum Gaussian kernel to increase the signal to noise ratio. Detailed procedures for the acquisition and processing of FDG-PET images have been described in a previous study (30).

Cells and reagents. Hippocampal cells were isolated as described previously (31) from 3xTg-AD mice and cultured in Dulbecco's modified Eagle's medium (Sigma-Aldrich; Merck KGaA;) supplemented with $10 \%$ fetal calf serum (Gibco; Thermo Fisher Scientific, Inc.). Hippocampal cells were cultured at $37^{\circ} \mathrm{C}$ for $12 \mathrm{~h}$ in a humidified atmosphere of $5 \% \mathrm{CO}_{2}$. 
mRNA expression analysis by reverse transcriptionquantitative polymerase chain reaction ( $R T-q P C R)$. Total cellular RNA was extracted from the hippocampal neurons of each group using RNeasy Mini kit (Qiagen Sciences, Inc., Gaithersburg, MD, USA) according to the manufacturer's protocol. A total of $1 \mu \mathrm{g}$ total RNA was reverse transcribed into cDNA. One-tenth of the cDNA was subjected to qPCR using an iQ SYBR Green system. All the primers were synthesized by Invitrogen (Thermo Fisher Scientific, Inc.) and indicated in Table I. PCR following thermocycling conditions were performed: 45 amplification cycles consisting of denaturation at $95^{\circ} \mathrm{C}$ for $5 \mathrm{~min}$, primer annealing at $66^{\circ} \mathrm{C}$ for $20 \mathrm{sec}$ with touchdown to $56^{\circ} \mathrm{C}$ for $20 \mathrm{sec}$, and applicant extension at $72^{\circ} \mathrm{C}$ for $5 \mathrm{~min}$. Relative levels of mRNA expression were calculated using the $2^{-\Delta \Delta C q}$ method (32). Results were expressed as a fold change relative to normal controls by comparing levels of target mRNA expression to that of the control group.

ELISA. Hippocampal neurons $\left(1 \times 10^{7}\right.$ cells) were prepared and lysed using cells lysis buffer (FNN0021; Thermo Fisher Scientific, Inc.). Expression levels of neprilysin (15-0106-42), insulin (PA5-29350), Abeta-42 (437900), Abeta-40 (KMB3441), interleukin (IL)-1 $\beta$ (LHC0011M), monocyte chemoattractant protein (MCP)-1 (88-7391-88), insulin-like growth factor binding protein (IGFBP)-3 (EMIGFBP3) and vascular endothelial growth factor (VEGF)- $\beta$ (MBS3205) were analyzed using ELISA kits (all Thermo Fisher Scientific, Inc.) according to the manufacturer's instrument.

Morphological analysis and terminal deoxynucleotidyl transferase-mediated dUTP nick end labeling (TUNEL) assay. Morphological of hippocampal neurons was analyzed by microscopy (Bx51; Olympus Corporation; Shinjuku-ku, Japan). Apoptotic neuronal cells in hippocampus in experimental mice were analyzed using TUNEL assay (DeadEnd ${ }^{\mathrm{TM}}$ Colorimetric Tunel system; Promega Corporation; Madison, WI, USA) according to the manufacturer's instructions. The slides were analyzed with fluorescence microscopy (magnification, x200; Bx51; Olympus Corporation).

Histological and immunohistochemical analysis. Immunohistochemical staining of neuroprotective proteins, namely forkhead box protein P2 (Foxp-2), SxIP motif-containing proteins (SxIPs) and end-binding proteins $(E B s)$, in the hippocampi of experimental mice was used to determine the effects of Simvastatin on neuronal loss. Staining was performed on the cerebral neurons in the hippocampi of randomly selected animals ( $n=3$ per group) in the Simvastatin and PBS-treated groups. Immunohistochemical procedures were performed as described previously (33). Briefly, the brains were frozen and coronal sections were cut in a cryostat. The tissues were cut into $4-\mu \mathrm{m}$ thick sections and mounted on glass slides. The paraffinized sections were heated in an oven at $65^{\circ}$ for $24 \mathrm{~h}$, dewaxed to water and rinsed with PBS three times. The washed sections were placed in EDTA buffer (Beinuo Bioscience Inc., Shanghai, China), and then boiled at a low heat following at $65^{\circ} \mathrm{C}$ for a total of three intervals $(10 \mathrm{~min}$ each). Following natural cooling, the sections were washed with PBS three times, and were placed into $3 \%$ hydrogen peroxide solution (Beina Bioscience Inc.), for incubation at room temperature for $10 \mathrm{~min}$, to block endogenous peroxidase. Free-floating sections were rinsed with PBS and placed in a solution containing primary mouse monoclonal antibodies directed against Foxp-2 (ABE73), SxIP (ABE86) and EB (ABC467; all 1:2,000 dilution; all EMD Millipore; Billerica, MA, USA) at $4^{\circ} \mathrm{C}$ overnight. After rinsing, sections were incubated for $1 \mathrm{~h}$ at room temperature with horseradish peroxidase (HRP)-conjugated goat anti-rabbit IgG mAb (1:5,000 dilution; PV-6001, ZSGB-BIO, Beijing, China). The sections were then washed with PBS and observed by fluorescent video microscopy (BZ-9000; Keyence Corporation, Osaka, Japan).

Western blotting. Hippocampal cells and cerebrospinal fluid were obtained as described previously (34), homogenized in lysate buffer containing protease inhibitor and centrifuged at $8,000 \mathrm{~g} / \mathrm{min}$ at $4^{\circ} \mathrm{C}$ for $10 \mathrm{~min}$. Total protein was extracted from the resulting supernatant using a Protein Extraction kit (20021; Qiagen Sciences, Inc., Gaithersburg, MD, USA), according to the manufacturer's instructions. SDS assays were performed as described previously (35). For western blot analysis, primary antibodies anti-bax (1:1,000; ab32503), anti-Bcl-2 (1:1,000; ab194583), anti-caspase-3 (1:1,000; ab13847), anti-caspase-9 (1:1,000; ab18571), anti-p38 (1:1,000; ab31828), anti-ERK (1:1,000; ab176660), anti $\beta$-actin (1:1,000; ab8226; all Abcam; Cambridge, UK) were added to PVDF membranes (EMD Millipore) and incubated at $4^{\circ} \mathrm{C}$ overnight. Membranes were blocked in $5 \%$ skimmed milk for $1 \mathrm{~h}$ at $37^{\circ} \mathrm{C}$ and then incubated with HRP-conjugated goat anti-rabbit $\operatorname{IgG}$ mAb (PV-6001; ZSGB-BIO) for $24 \mathrm{~h}$ at $4^{\circ} \mathrm{C}$. A Ventana Benchmark automated staining system was used to determine protein expression in tumor tissues (Olympus BX51; Olympus Corporation).

Statistical analysis. All data are represented as the mean \pm standard error $(n=3)$. Statistical significance was determined using a two-tailed Student's t-test followed by two-way analysis of variance (ANOVA), a Kaplan-Meier test or one-way analysis of variance (ANOVA). Statistical analysis was performed using GraphPad software 5.0 (GraphPad Software, Inc., La Jolla, CA, USA) and $\mathrm{P}<0.05$ was considered to indicate a statistically significant difference.

\section{Results}

Simvastatin inhibits hippocampal cells apoptosis. Reducing the apoptosis of hippocampal neurons is a key requirement of therapeutics for Alzheimer's disease. To access the therapeutic effects of Simvastatin in Alzheimer's disease, hippocampal cells from 3xTg-AD mice were cultured and treated with Simvastatin or PBS (Control). After incubation with PBS, hippocampal cells exhibited shrinkage, fragmentation into membrane-bound apoptotic bodies and phagocytosis of neighboring cells, while treatment with Simvastatin suppressed these phenomena (Fig. 1A). As depicted in Fig. 1B, the apoptotic rate of hippocampal cells was significantly decreased following treatment with Simvastatin $(10 \mathrm{mg} / \mathrm{ml})$, relative to controls $(\mathrm{P}<0.01)$. In addition, levels of apoptotic protein expression in the hippocampal cells were evaluated following treatment with Simvastatin. As depicted in Fig. 1C, it was observed that Simvastatin treatment significantly increased the expression of Bax and Bcl- 2 in vitro, when compared to the 
Table I. Sequences of primers were used in this study.

Sequence

\begin{tabular}{lll}
\cline { 2 - 3 } Gene name & \multicolumn{1}{c}{ Reverse } & \multicolumn{1}{c}{ Forward } \\
\hline Bax & 5'-CCTGTGCACCAAGGTGCCGGAACT-3' & 5'-CCACCCTGGTCTTGGATCCAGCCC-3' \\
Bcl-2 & 5'-TTGTGGCCTTCTTTGAGTTCGGTG-3' & 5'-GGTGCCGGTTCAGGTACTCAGTCA-3' \\
Caspase-3 & 5'-TCTGACGAATCTCCTCCAC-3' & 5'-AAAGTTTTCAATGACCAAGC-3' \\
Caspase-8 & 5'-AGTCTATTTTATTATGGGCTCG-3' & 5'-TGGATGTTTATGTCACCTTTTC-3' \\
$\beta$-actin & 5'-CGGAGTCAACGGATTTGGTC-3' & 5'-AGCCTTCTCCATGGTCGTGA-3' \\
\hline
\end{tabular}

Bcl-2, B-cell lymphoma 2; Bax, Bcl-2 associated X protein.

A

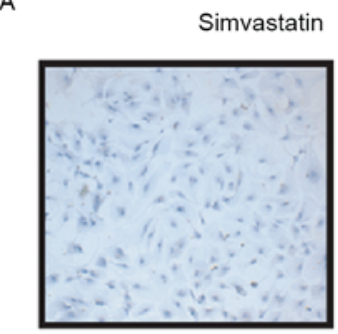

C

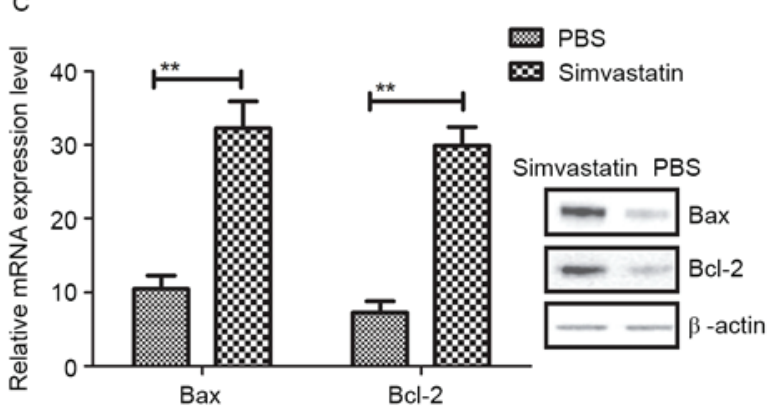

PBS

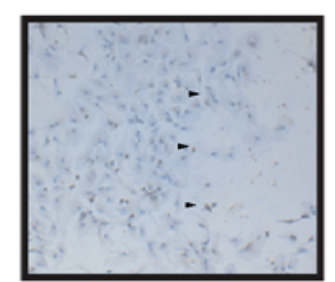

Bcl-2

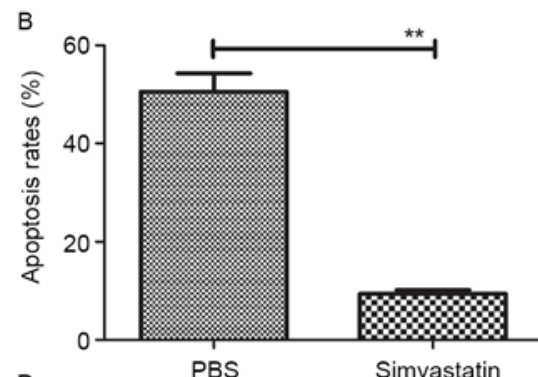

D

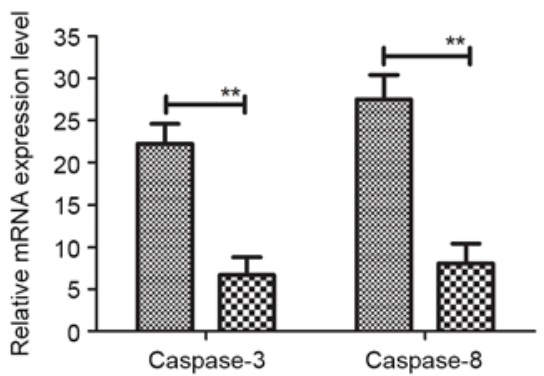

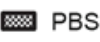

$\infty$ Simvastatin
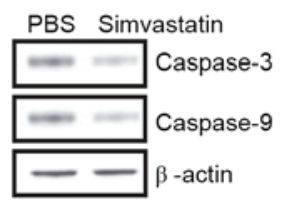

Figure 1. Apoptosis of hippocampal cells following treatment with Simvastatin. (A) Morphological analysis of hippocampal cells following treatment with Simvastatin or PBS. Magnification, x200. Arrows indicated that hippocampal cells exhibited shrinkage, fragmentation into membrane-bound apoptotic bodies and phagocytosis of neighboring cells. (B) Apoptosis of hippocampal cells following Simvastatin or PBS treatment assessed by a TUNEL assay (C) Expression of the anti-apoptotic genes Bcl-2 and Bax in hippocampal cells following treatment with Simvastatin or PBS. (D) Expression of the pro-apoptotic genes caspase-3 and caspase-8 in hippocampal cells following treatment with Simvastatin or PBS. All data are represented as the mean \pm standard error of the mean ( $\mathrm{n}=3$ per group). ${ }^{* * *} \mathrm{P}<0.01$ vs. PBS (control). PBS, phosphate-buffered saline; Bcl-2, B-cell lymphoma protein; Bax, Bcl-2-associated X protein.

control group (both $\mathrm{P}<0.01$ ). In addition, levels of caspase- 8 and caspase-3 were significantly lower in hippocampal cells following Simvastatin treatment, relative to controls (both $\mathrm{P}<0.01$; Fig. 1D). These results suggest that Simvastatin may reduce apoptosis by regulating the activation of apoptotic genes.

Simvastatin regulates extracellular signal-regulated kinase (ERK)/MAPK signaling in vitro and in vivo. A previous study demonstrated that p38 MAPK induced cell apoptosis via the mitochondrial pathway (36). In addition, it has been observed that ERK/MAPK activation in the prefrontal cortex reversed early memory deficit, and that this effect was downregulated in a mouse model of Alzheimer's disease (37). Furthermore, ERK/MAPK induced the mitochondrial pathway of apoptosis through regulating the expression of associated apoptotic factors, including caspase-9, Bcl-2 and
Bax (38). Therefore, the expression and phosphorylation of ERK/MAPK were evaluated in hippocampal cells. As depicted in Fig. 2A, the expression and phosphorylation of p38 MAPK were markedly elevated in the Simvastatin group compared to the control. By contrast, levels of ERK expression and phosphorylation were notably reduced following treatment with Simvastatin (Fig. 2B). In addition, the pattern of activated ERK/MAPK in 3xTg-AD model mice was evaluated 28 days after treatment with Simvastatin. As depicted in Fig. 2C, Simvastatin-treated mice exhibited significantly increased levels of phospho-ERK/MAPK in the dorsal gyrus of the hippocampus, relative to the control group $\left({ }^{* *} \mathrm{P}<0.01\right)$. This result was verified by immunohistochemistry (Fig. 2D). Collectively, these data suggest that Simvastatin regulates ERK/MAPK signaling, leading to an increase expression levels of phospho-ERK/MAPK in the dorsal gyrus both in vitro and in vivo. 
A

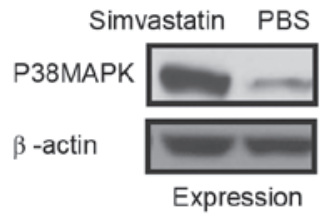

C

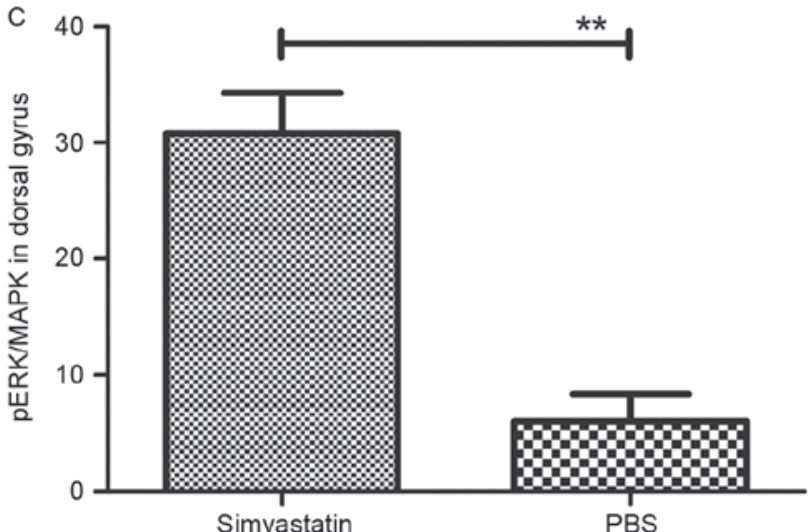

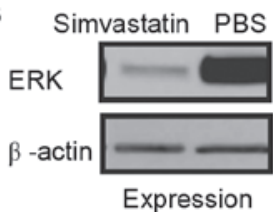

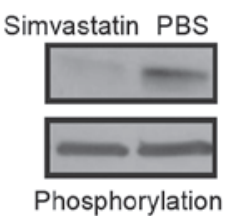

D
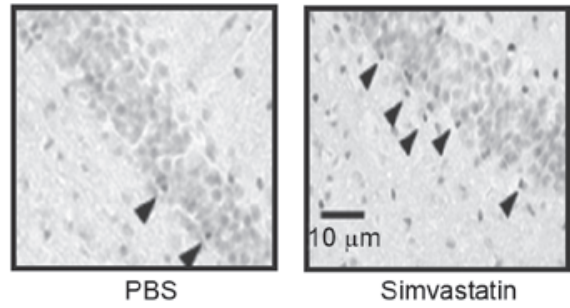

Figure 2. Regulation of ERK/MAPK signaling in hippocampal cells by Simvastatin. (A) Levels of p38 MAPK expression and phosphorylation in hippocampal cells following treatment with Simvastatin or PBS. (B) Levels of ERK expression and phosphorylation in hippocampal cells following treatment with Simvastatin or PBS. (C) Level of pERK/MAPK in the hippocampal dorsal gyrus of mice with Alzheimer's disease following treatment with Simvastatin or PBS. ${ }^{* *} \mathrm{P}<0.01$ vs. control. (D) Immunohistochemistry of the hippocampal dorsal gyrus. Arrows indicated pERK/MAPK levels in dorsal gyrus of hippocampus. Data are represented as the mean \pm standard error of the mean ( $n=3$ per group). PBS, phosphate-buffered saline; ERK, extracellular signal-regulated protein kinase; p38 MAPK, p38 mitogen-activated protein kinase.

Simvastatin attenuates the expression of pathological factors in 3xTg-AD model mice. It has been documented that pathological agents are present in patients with Alzheimer's disease (39). Therefore, the expression of pathological factors, namely Abeta-40, Abeta-42, IL-1 $\beta$, MCP-1, neprilysin, insulin, IGFBP-3 and VEGF- $\beta$, were evaluated in 3xTg-AD model mice following treatment with Simvastatin. As depicted in Fig. 3A, it was observed that Simvastatin treatment significantly reduced intracellular levels of Abeta-42 and Abeta-40 peptides in the cerebrospinal fluid (both $\mathrm{P}<0.01$ vs. control). Levels of IL-1 $\beta$ and MCP-1 expression were also significantly decreased in the cerebrospinal fluid following treatment with Simvastatin, relative to the PBS group (both $\mathrm{P}<0.01$; Fig. $3 \mathrm{~B}$ ). In addition, levels of neprilysin and insulin were analyzed in the Alzheimer's disease mice. As depicted in Fig. 3C, it was observed that neprilysin and insulin were significantly upregulated following Simvastatin treatment (both $\mathrm{P}<0.01$ vs. control). Furthermore, Simvastatin treatment significantly downregulated the expression of IGFBP-3 and VEGF- $\beta$ (both $\mathrm{P}<0.01$ vs. control), as determined by ELISA (Fig. 3D). Collectively, these data suggest that Simvastatin inhibits the expression of harmful pathological factors and promotes the expression of beneficial pathological factors in the cerebrospinal fluid of 3xTg-AD mice.

Simvastatin has beneficial effects on the cognitive competence of the hippocampal network. To determine the effects of Simvastatin on network excitability, hippocampal slices from non-treated and Simvastatin-treated 3xTg-AD mice were scored to access the outcomes of treatment. As depicted in Fig. 4A, compared with pretreatment, the degree of dementia significantly improved after 16 days of treatment with Simvastatin $(\mathrm{P}<0.05)$ and the improvement gradually increased after 22 days $(\mathrm{P}<0.01)$ and 27 days $(\mathrm{P}<0.001)$. Immunohistochemical analysis of the hippocampus also indicated that Foxp-2, SxIP and EB were upregulated in the brain of Simvastatin-treated mice (Fig. 4B). To determine the long-term activity of Simvastatin, the survival rates of mice treated with or without Simvastatin were calculated following a 36-month observation period. It was observed that survival rate was significantly higher in Simvastatin-treated mice compared with controls $(\mathrm{P}<0.01$; Fig $4 \mathrm{C})$. In addition, PET scans of the hippocampi in the Simvastatin and control groups exhibited marked differences in the dispersion of the pyramidal cell layer (Fig. 4D). Furthermore, the cognitive competence and anxiety of 3xTg-AD mice were evaluated after 28 days of treatment with Simvastatin. Morris water maze and open field tests indicated that cognitive competence was significantly improved by Simvastatin treatment $(\mathrm{P}<0.01$; Fig. 4E and F). Collectively, these data indicate that Simvastatin has significant and fast-acting effects on circuit excitability, neuroprotective protein levels and cognitive competence in vivo, potentially due to the inhibitory effects of Simvastatin on neuronal apoptosis.

\section{Discussion}

The majority of senile dementia is caused by vascular dementia and results in a decreased quality of life (40). The combined effects of vascular dementia and Alzheimer's disease cause greater damage to the nervous system and impair cognitive ability in elderly patients with senile dementia (41). A previous 
A
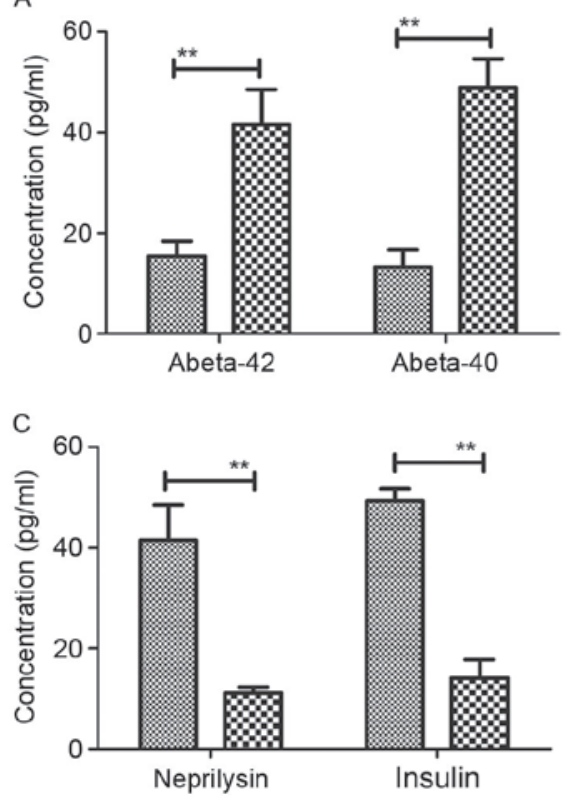

B
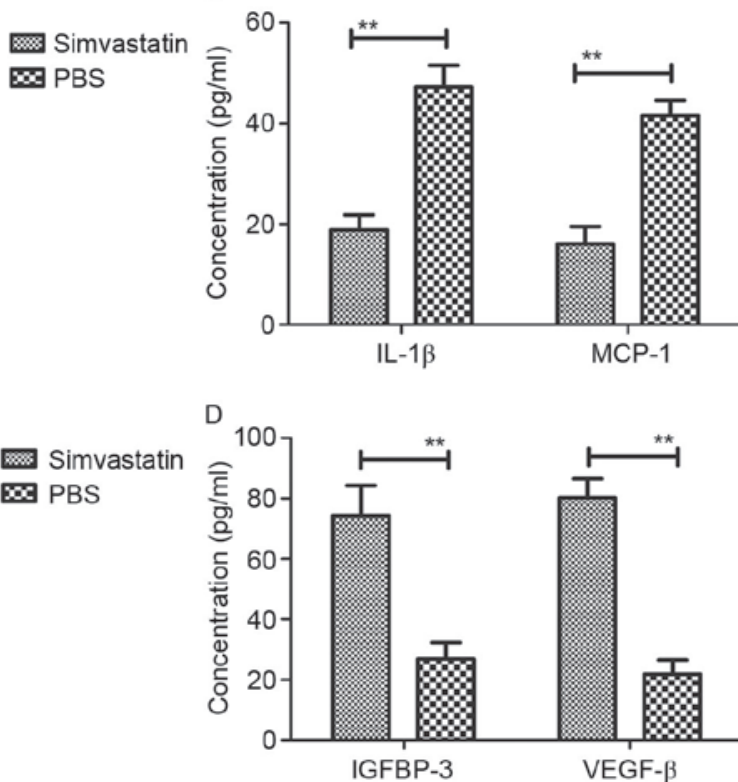

D

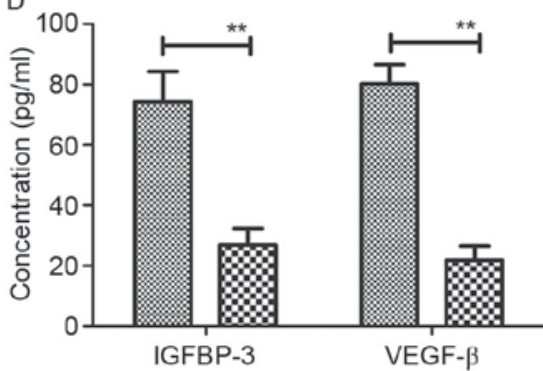
$\infty$ PBS

Figure 3. Expression of pathological factors in 3xTg-AD model mice following treatment with Simvastatin. ELISA assays were performed and determine that (A) Simvastatin treatment reduced intracellular levels of Abeta-42 and Abeta-40 peptides in the cerebrospinal fluid. Furthermore, (B) Simvastatin treatment downregulated IL-1 $\beta$ and MCP-1 in the cerebrospinal fluid and (C) Simvastatin treatment upregulated neprilysin and insulin in the cerebrospinal fluid. Additonally, (D) Simvastatin treatment upregulated IGFBP-3 and VEGF- $\beta$ in the cerebrospinal fluid. Data are represented as the mean \pm standard error of the mean ( $\mathrm{n}=3$ per group). ${ }^{* *} \mathrm{P}<0.01$ vs. control. PBS, phosphate-buffered saline; 3xTg-AD, triple-transgenic Alzheimer's disease; Abeta, amyloid beta; IL, interleukin; MCP, monocyte chemoattractant protein; IGFBP, insulin-like growth factor-binding protein; VEGF, vascular endothelial growth factor.
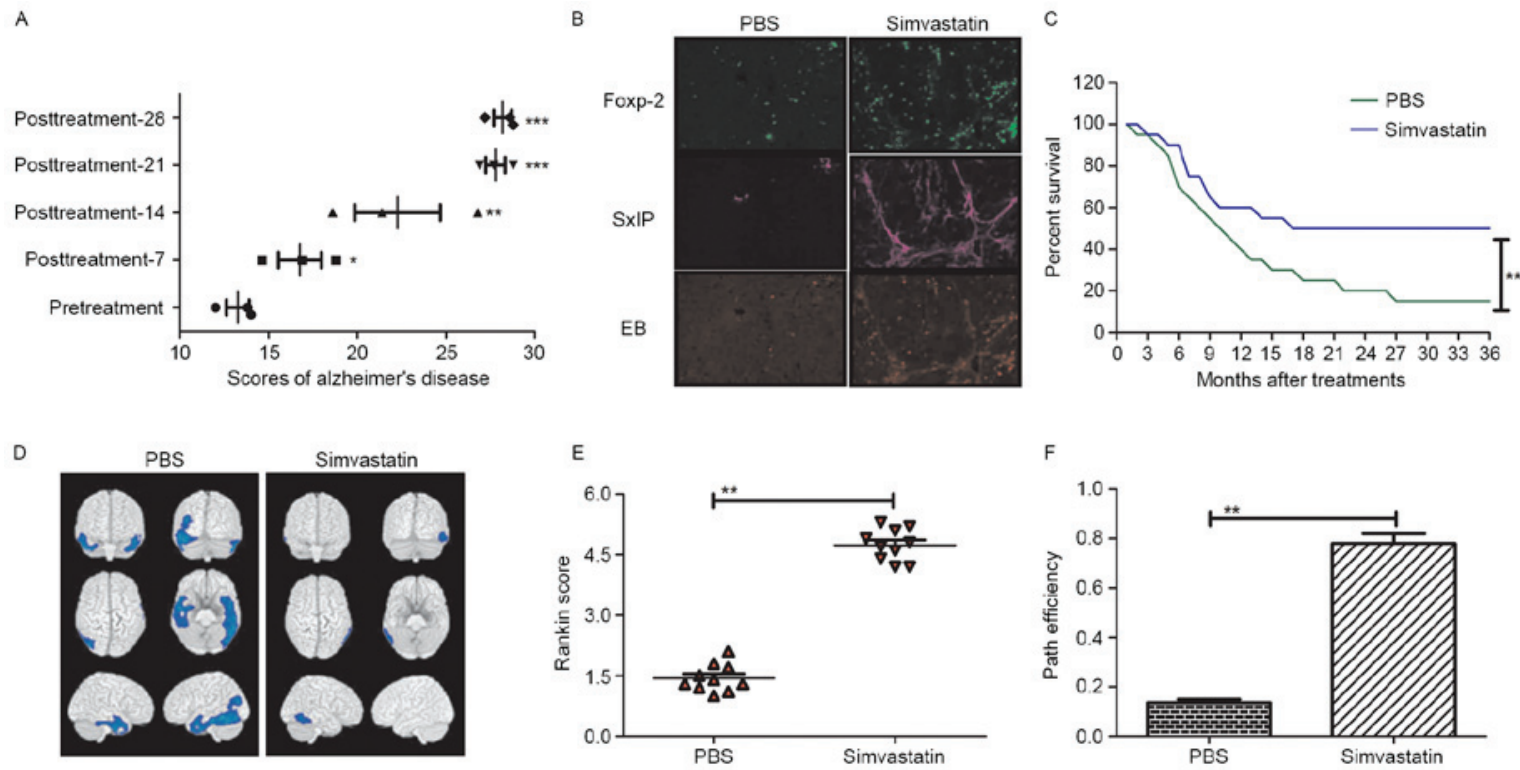

Figure 4. Therapeutic effects of Simvastatin in mice with Alzheimer's disease. (A) Therapeutic effects of Simvastatin on Alzheimer's disease over a 30-day treatment period. ${ }^{*} \mathrm{P}<0.05,{ }^{* *} \mathrm{P}<0.01$ and ${ }^{* * * *} \mathrm{P}<0.001$ vs. pretreatment value. (B) The neuroprotective proteins Foxp-2, EB and SxIP were upregulated in the hippocampal cells of mice with Alzheimer's disease following Simvastatin treatment. (C) Simvastatin treatment significantly improved the survival rate of Alzheimer's disease mice compared to the PBS group. ${ }^{* *} \mathrm{P}<0.01$ vs. control. (D) Hippocampal excitability was reduced after 28 days of treatment with Simvastatin compared to the PBS group $\left({ }^{* *} \mathrm{P}<0.01\right.$; Tukey HSD test). (E) the Morris water maze conducted on mice indicated that Simvastatin treatment significantly lowered prospective observations of Alzheimer's disease, as determined by Rankin scoring. (F) Open field tests indicated that Simvastatin treatment significantly improved cognitive competence, as determined by path efficiency measures. Data are represented as the mean \pm standard error of the mean ( $\mathrm{n}=3$ per group) and the data for the survival rate are represented as mean of triple trials.

study indicated that hippocampal apoptosis was correlated with hypoxia and ischemia, and brain injury during chronic cerebral hypoperfusion, as indicated by increases in infarct volume, was typically sustained (42). Chronic cerebral hypoperfusion is the most prevalent cause of Alzheimer's disease, and modeling of chronic cerebral hypoperfusion through surgical procedures, such as bilateral carotid artery ligation, may aid to elucidate the mechanism of Alzheimer's disease in animal models (43). 
Alzheimer's disease is the most prevalent senile disease to occur in the elderly. In addition, aneurysmal subarachnoid hemorrhage in patients with Alzheimer's disease affects patient prognosis and the occurrence of various complications (44). In the majority of adult patients with Alzheimer's disease and associated cognitive disorder, the mechanisms and etiology of cognitive disorder remain unknown (45). The current preclinical study demonstrated that Simvastatin was effective in inhibiting the apoptosis of hippocampal cells and inflammation in mice with Alzheimer's disease. Simvastatin exerts neuroprotective effects though competitive binding to HMG CoA reductase, and modulates inflammation and augments cerebral blood flow following its administration $(46,47)$. However, a previous study has suggested that the therapeutic effects of Simvastatin in preventing cerebral vasospasm are controversial and warrant verification (48).

Numerous therapeutic trials for Alzheimer's disease have been unsuccessful $(49,50)$. Disease-modifying treatments and the condition for Alzheimer's disease have progress and aim to impact cognition, function, lifespan and healthcare. Thus, Alzheimer's disease remains difficult to manage for pathologists and clinicians (51). However, few patients with Alzheimer's disease exhibit a positive response to drug therapy. The clinical outcomes of Alzheimer's disease greatly impact on patient quality of life. In addition, the incidence of Alzheimer's disease has recently increased, potentially due to deteriorations in food and the environment (52). The effects of Simvastatin in the treatment of Alzheimer's disease are not well studied, and to the best of our knowledge, no previous study has assessed the effects of $100 \mathrm{mg}$ Simvastatin $(53,54)$. In the present study, high-dose Simvastatin $(100 \mathrm{mg} / \mathrm{kg})$ was administered to mice in an attempt to cure Alzheimer's disease, where it was found to improve symptoms. This may have been due to the effects of high-dose Simvastatin in preventing hippocampal cell apoptosis, as a process typically linked with the occurrence of Alzheimer's disease.

In the current study, 30-day treatment with high-dose Simvastatin resulted in improved clinical outcomes and cognition in mice with Alzheimer's disease. Improvements in apoptosis and cognition were consistent with the results of a recent study, indicating that Simvastatin had regulatory effects on the expression of pathological factors associated with Alzheimer's disease, enabling it to achieve positive therapeutic outcome (55-57).

In conclusion, the present study suggested that Simvastatin treatment was well-tolerated in mice with Alzheimer's disease. Simvastatin potentially exerted inhibitory effects on the apoptosis of hippocampal neurons, thus aiding to recover memory deficit. In addition, Simvastatin may have regulated ERK/MAPK signaling pathways, which have previously been associated with the nervous system by regulation of memory deficits (24). Collectively, these results indicate that Simvastatin may be a viable drug therapy in the treatment of Alzheimer's disease.

\section{References}

1. Cheng X, Zhang L and Lian YJ: Molecular targets in Alzheimer's disease: From pathogenesis to therapeutics. Biomed Res Int 2015: 760758, 2015.
2. Stella F, Laks J, Govone JS, de Medeiros K and Forlenza OV: Association of neuropsychiatric syndromes with global clinical deterioration in Alzheimer's disease patients. Int Psychogeriatr 28: 779-786, 2016.

3. Garcia KO, Ornellas FL, Martin PK, Patti CL, Mello LE, Frussa-Filho R, Han SW and Longo BM: Therapeutic effects of the transplantation of VEGF overexpressing bone marrow mesenchymal stem cells in the hippocampus of murine model of Alzheimer's disease. Front Aging Neurosci 6: 30, 2014.

4. Ramos B, Baglietto-Vargas D, del Rio JC, Moreno-Gonzalez I, Santa-Maria C, Jimenez S, Caballero C, Lopez-Tellez JF, Khan ZU, Ruano D, et al: Early neuropathology of somatostatin/NPY GABAergic cells in the hippocampus of a PS1xAPP transgenic model of Alzheimer's disease. Neurobiol Aging 27: 1658-1672, 2006.

5. Youn H, Ji I, Ji HP, Markesbery WR and Ji TH: Under-expression of Kalirin-7 increases iNOS activity in cultured cells and correlates to elevated iNOS activity in Alzheimer's disease hippocampus. J Alzheimers Dis 12: 271-281, 2007.

6. Namba T, Maekawa M, Yuasa S, Kohsaka S and Uchino S: The Alzheimer's disease drug memantine increases the number of radial glia-like progenitor cells in adult hippocampus. Glia 57: 1082-1090, 2009.

7. Obulesu M and Lakshmi MJ: Apoptosis in Alzheimer's disease: An understanding of the physiology, pathology and therapeutic avenues. Neurochem Res 39: 2301-2312, 2014.

8. Turunc Bayrakdar E, Uyanikgil Y, Kanit L, Koylu E and Yalcin A: Nicotinamide treatment reduces the levels of oxidative stress, apoptosis, and PARP-1 activity in A $\beta(1-42)$-induced rat model of Alzheimer's disease. Free Radic Res 48: 146-158, 2014.

9. Bartolomé F, Muñoz U, Esteras N, Alquezar C, Collado A, Bermejo-Pareja F and Martín-Requero A: Simvastatin overcomes the resistance to serum withdrawal-induced apoptosis of lymphocytes from Alzheimer's disease patients. Cell Mol Life Sci 67: 4257-4268, 2010.

10. Absalon S, Kochanek DM, Raghavan V and Krichevsky AM: MiR-26b, upregulated in Alzheimer's disease, activates cell cycle entry, tau-phosphorylation, and apoptosis in postmitotic neurons. J Neurosci 33: 14645-14659, 2013.

11. Jendresen C, Arskog V, Daws MR and Nilsson LN: The Alzheimer's disease risk factors apolipoprotein E and TREM2 are linked in a receptor signaling pathway. J Neuroinflammation 14: 59, 2017.

12. Jiang L, Tang $Y$ and Huang $X$ : Brain cell apoptosis after cerebral hypoxia-ischemia in neonatal rat. Zhonghua Yi Xue Za Zhi 78: 567-569, 1998 (In Chinese).

13. Zhang XQ, Zhang ZM, Yin XL, Zhang K, Cai H and Ling F: Exploring the optimal operation time for patients with hypertensive intracerebral hemorrhage: Tracking the expression and progress of cell apoptosis of prehematomal brain tissues. Chin Med J (Engl) 123: 1246-1250, 2010.

14. Johnson S, Tazik S, Lu D, Johnson C, Youdim MB, Wang J, Rajkowska G and Ou XM: The new inhibitor of monoamine oxidase, M30, has a neuroprotective effect against dexamethasone-induced brain cell apoptosis. Front Neurosci 4: 180, 2010.

15. Yang S, Zhou G, Liu H, Zhang B, Li J, Cui R and Du Y: Protective effects of p38 MAPK inhibitor SB202190 against hippocampal apoptosis and spatial learning and memory deficits in a rat model of vascular dementia. Biomed Res Int 2013: 215798, 2013.

16. Yürüker V, Nazıroğlu M and Senol N: Reduction in traumatic brain injury-induced oxidative stress, apoptosis, and calcium entry in rat hippocampus by melatonin: Possible involvement of TRPM2 channels. Metab Brain Dis 30: 223-231, 2015.

17. Chatterjee A, Chattopadhyay D and Chakrabarti G: MiR-16 targets Bcl-2 in paclitaxel-resistant lung cancer cells and overexpression of miR-16 along with miR-17 causes unprecedented sensitivity by simultaneously modulating autophagy and apoptosis. Cell Signal 27: 189-203, 2015.

18. Liu Q and Gazitt Y: Potentiation of dexamethasone-, paclitaxel-, and Ad-p53-induced apoptosis by Bcl-2 antisense oligodeoxynucleotides in drug-resistant multiple myeloma cells. Blood 101: 4105-4114, 2003

19. Chen X, Wang W, Zhang L, He Z, Cai Q, Liu X, Cheng C and Wu L: Effects of Ad-p27mt gene transfer on the expression of Bax, Bcl-2, VEGF and MMP-9 in the transplanted liver tumors in nude mice. J Huazhong Univ Sci Technolog Med Sci 30: 611-614, 2010.

20. Nichol KE, Kim R and Cotman CW: Bcl-2 family protein behavior in frontotemporal dementia implies vascular involvement. Neurology 56 (11 Suppl 4): S35-S40, 2001. 
21. Pan Y, Dai T and Yang Q: Influence of aniracetam on the learning ability, memory ability and the expression of BCL-2 of hippocampal in vascular dementia model rats. Zhongguo Ying Yong Sheng Li Xue Za Zhi 25: 180-181, 227, 2009 (In Chinese).

22. Aboutaleb N, Shamsaei N, Khaksari M, Erfani S, Rajabi H and Nikbakht F: Pre-ischemic exercise reduces apoptosis in hippocampal CA3 cells after cerebral ischemia by modulation of the $\mathrm{Bax} / \mathrm{Bcl}-2$ proteins ratio and prevention of caspase-3 activation. J Physiol Sci 65: 435-443, 2015.

23. Chen T, Wang C, Sha S, Zhou L, Chen L and Chen L: Simvastatin enhances spatial memory and long-term potentiation in hippocampal CA1 via upregulation of $\alpha 7$ nicotinic acetylcholine receptor. Mol Neurobiol 53: 4060-4072, 2016.

24. Ramos MC, Sierra S, Ramirez C, Velasco J and Burgos JS: Simvastatin modulates the Alzheimer's disease-related gene seladin-1. J Alzheimers Dis 28: 297-301, 2012.

25. Longenberger J and Shah ZA: Simvastatin and other HMG-CoA reductase inhibitors on brain cholesterol levels in Alzheimer's disease. Curr Alzheimer Res 8: 434-442, 2011

26. Sarazin M, Lagarde J and Bottlaender M: Distinct tau PET imaging patterns in typical and atypical Alzheimer's disease. Brain 139: 1321-1324, 2016.

27. TurelOandBechara A:Atriadicreflective-impulsive-interoceptive awareness model of general and impulsive information system use: Behavioral tests of neuro-cognitive theory. Front Psychol 7: $601,2016$.

28. Nunez J: Morris water maze experiment. J Vis Exp: pii: 897, 2008.

29. López-Cancio E, Salvat M, Cerdà N, Jiménez M, Codas J, Llull L, Boned S, Cano LM, Lara B, Molina C, et al: Phone and video-based modalities of central blinded adjudication of modified rankin scores in an endovascular stroke trial. Stroke 46: 3405-3410, 2015.

30. Teoh EJ, McGowan DR, Bradley KM, Belcher E, Black E, Moore A, Sykes A and Gleeson FV: 18F-FDG PET/CT assessment of histopathologically confirmed mediastinal lymph nodes in non-small cell lung cancer using a penalised likelihood reconstruction. Eur Radiol 26: 4098-4106, 2016.

31. Long Y, Wang Y, Ji G, Yan L, Hu F and Gu A: Neurotoxicity of perfluorooctane sulfonate to hippocampal cells in adult mice. PLoS One 8: e54176, 2013.

32. Peng R, Zhai Y, Ding H, Di T, Zhang T, Li B, Shen W and Wei Z: Analysis of reference gene expression for real-time PCR based on relative quantitation and dual spike-in strategy in the silkworm Bombyx mori. Acta Biochim Biophys Sin (Shanghai) 44 614-622, 2012.

33. Dirani M, Nasreddine W, Abdulla F and Beydoun A: Seizure control and improvement of neurological dysfunction in Lafora disease with perampanel. Epilepsy Behav Case Rep 2: 164-166, 2014.

34. Leasure JL and Decker L: Social isolation prevents exercise-induced proliferation of hippocampal progenitor cells in female rats. Hippocampus 19: 907-912, 2009.

35. Wai-Hoe L, Wing-Seng L, Ismail Z and Lay-Harn G: SDS-PAGE-based quantitative assay for screening of kidney stone disease. Biol Proced Online 11: 145-160, 2009.

36. Srivastava RK: Intracellular mechanisms of TRAIL and its role in cancer therapy. Mol Cell Biol Res Commun 4: 67-75, 2000.

37. Feld M, Krawczyk MC, Sol Fustiñana M, Blake MG, Baratti CM, Romano A and Boccia MM: Decrease of ERK/MAPK overactivation in prefrontal cortex reverses early memory deficit in a mouse model of Alzheimer's disease. J Alzheimers Dis 40: 69-82, 2014.

38. Miglietta A, Bozzo F, Bocca C, Gabriel L, Trombetta A, Belotti S and Canuto RA: Conjugated linoleic acid induces apoptosis in MDA-MB-231 breast cancer cells through ERK/MAPK signalling and mitochondrial pathway. Cancer Lett 234: 149-157, 2006.

39. Maltsev AV, Santockyte R, Bystryak S and Galzitskaya OV: Activation of neuronal defense mechanisms in response to pathogenic factors triggering induction of amyloidosis in Alzheimer's disease. J Alzheimers Dis 40: 19-32, 2014.

40. Venketasubramanian N, Sahadevan S, Kua EH, Chen CP and Ng TP: Interethnic differences in dementia epidemiology: Global and Asia-Pacific perspectives. Dement Geriatr Cogn Disord 30: 492-498, 2010.
41. Battistin L and Cagnin A: Vascular cognitive disorder. A biological and clinical overview. Neurochem Res 35: 1933-1938, 2010.

42. Sekhon LH, Morgan MK, Spence I and Weber NC: Chronic cerebral hypoperfusion: Pathological and behavioral consequences. Neurosurgery 40: 548-556, 1997.

43. Philbert MA, Billingsley ML and Reuhl KR: Mechanisms of injury in the central nervous system. Toxicol Pathol 28: 43-53, 2000 .

44. Takizawa C, Gemmell E, Kenworthy J and Speyer R: A systematic review of the prevalence of oropharyngeal dysphagia in stroke, parkinson's disease, alzheimer's disease, head injury, and pneumonia. Dysphagia 31: 434-441, 2016.

45. Shams S and Wahlund LO: Cerebral microbleeds as a biomarker in Alzheimer's disease? A review in the field. Biomark Med 10: 9-18, 2016.

46. Endres M, Laufs U, Huang Z, Nakamura T, Huang $P$, Moskowitz MA and Liao JK: Stroke protection by 3-hydroxy-3-methylglutaryl (HMG)-CoA reductase inhibitors mediated by endothelial nitric oxide synthase. Proc Natl Acad Sci USA 95: 8880-8885, 1998.

47. Rossoni LV, Wareing M, Wenceslau CF, Al-Abri M, Cobb C and Austin C: Acute simvastatin increases endothelial nitric oxide synthase phosphorylation via AMP-activated protein kinase and reduces contractility of isolated rat mesenteric resistance arteries. Clin Sci (Lond) 121: 449-458, 2011.

48. Woo SW, Kim JH, Kang HI, Kim DR, Moon BG and Kim JS: High-dose simvastatin is effective in preventing cerebral vasospasm after aneurysmal subarachnoid hemorrhage: A prospective cohort study in Korean patients. J Korean Neurosurg Soc 58: 328-333, 2015.

49. Godyń J, Jończyk J, Panek D and Malawska B: Therapeutic strategies for Alzheimer's disease in clinical trials. Pharmacol Rep 68: 127-138, 2016.

50. Jia Q, Deng Y and Qing H: Potential therapeutic strategies for Alzheimer's disease targeting or beyond $\beta$-amyloid: Insights from clinical trials. Biomed Res Int 2014: 837157, 2014.

51. Cavaleri F: Review of amyotrophic lateral sclerosis, parkinson's and Alzheimer's diseases helps further define pathology of the novel paradigm for Alzheimer's with heavy metals as primary disease cause. Med Hypotheses 85: 779-790, 2015.

52. Manabe T, Mizukami K, Akatsu H, Teramoto S, Yamaoka K, Nakamura S, Ohkubo T, Kudo K and Hizawa N: Influence of pneumonia complications on the prognosis of patients with autopsy-confirmed Alzheimer's disease, dementia with Lewy bodies, and vascular dementia. Psychogeriatrics 16: 305-314, 2016.

53. Hoglund K, Thelen KM, Syversen S, Sjogren M, von Bergmann K, Wallin A, Vanmechelen E, Vanderstichele H, Lutjohann D and Blennow K: The effect of simvastatin treatment on the amyloid precursor protein and brain cholesterol metabolism in patients with Alzheimer's disease. Dement Geriatr Cogn Disord 19: 256-265, 2005.

54. Sala SG, Muñoz U, Bartolomé $F$, Bermejo $F$ and Martín-Requero A: HMG-CoA reductase inhibitor simvastatin inhibits cell cycle progression at the G1/S checkpoint in immortalized lymphocytes from Alzheimer's disease patients independently of cholesterol-lowering effects. J Pharmacol Exp Ther 324: 352-359, 2008.

55. de Azevedo AM and Goldberg Tabak D: Life-threatening capillary leak syndrome after G-CSF mobilization and collection of peripheral blood progenitor cells for allogeneic transplantation. Bone Marrow Transplant 28: 311-312, 2001.

56. Huang W, Li Z, Zhao L and Zhao W: Simvastatin ameliorate memory deficits and inflammation in clinical and mouse model of Alzheimer's disease via modulating the expression of miR-106b. Biomed Pharmacother 92: 46-57, 2017.

57. Jeong JH, Yum KS, Chang JY, Kim M, Ahn JY, Kim S, Lapchak PA and Han MK: Dose-specific effect of simvastatin on hypoxia-induced HIF-1 $\alpha$ and BACE expression in Alzheimer's disease cybrid cells. BMC Neurol 15: 127, 2015.

This work is licensed under a Creative Commons Attribution-NonCommercial-NoDerivatives 4.0 International (CC BY-NC-ND 4.0) License. 